\title{
Veering triangulations admit strict angle structures
}

\author{
CRAIG D HodgSON \\ J HYAM RUBiNSTEIN \\ HENRY SEGERMAN \\ STEPHAN TILLMANN
}

\begin{abstract}
Agol recently introduced the concept of a veering taut triangulation of a 3-manifold, which is a taut ideal triangulation with some extra combinatorial structure. We define the weaker notion of a "veering triangulation" and use it to show that all veering triangulations admit strict angle structures. We also answer a question of Agol, giving an example of a veering taut triangulation that is not layered.
\end{abstract}

$57 \mathrm{M} 50$

\section{Introduction}

A basic question in 3-dimensional topology is to relate the combinatorics of a triangulation of a 3-manifold to the geometry of the manifold. The work of Guéritaud and Futer [6] deals with the case of hyperbolic structures on once-punctured torus bundles and complements of two-bridge knots and links. They study the angle structures on the natural layered ideal triangulations of these manifolds, and use the volume maximization approach of Casson and Rivin to show that these triangulations are geometric, ie realized by positively oriented ideal hyperbolic tetrahedra.

In this paper, we introduce a new class of "veering triangulations," which includes the veering taut triangulations of Agol [1] and, in particular, the layered triangulations of once-punctured torus bundles. Our main result shows that these veering triangulations admit strict angle structures.

Throughout this section, $M$ will denote the interior of an orientable 3-manifold with boundary a disjoint union of tori, imbued with a fixed ideal triangulation.

Definition 1.1 (Taut angle structure) An angle-taut tetrahedron is an ideal tetrahedron equipped with an assignment of angles taken from $\{0, \pi\}$ to its edges so that two opposite edges are assigned $\pi$ and the other four are assigned 0 . A taut angle structure on $M$ is an assignment of angles taken from $\{0, \pi\}$ to the edges of each tetrahedron in $M$, such that every tetrahedron is angle-taut and the sum of all angles around each edge in $M$ is $2 \pi$. 
The choice of adjective taut for these angle structures is standard, but slightly unfortunate. Lackenby [10] introduced the following notion of a taut ideal triangulation in analogy with a taut foliation.

Definition 1.2 (Taut structure) A taut tetrahedron is an ideal tetrahedron with a coorientation assigned to each face, such that precisely two faces are cooriented into the tetrahedron, and precisely two are cooriented outwards. Each edge of a taut tetrahedron is assigned an angle of either $\pi$ if the coorientations on the adjacent faces agree, or 0 if they disagree. See Figure 1(a) for the only possible configuration (up to symmetry). An ideal triangulation of $M$ is taut if there is a coorientation assigned to each ideal triangle, such that every ideal tetrahedron is taut, and the sum of all angles around each edge in $M$ is $2 \pi$ (see Figure 1(b)). This will also be called a taut structure on $M$.

(a)

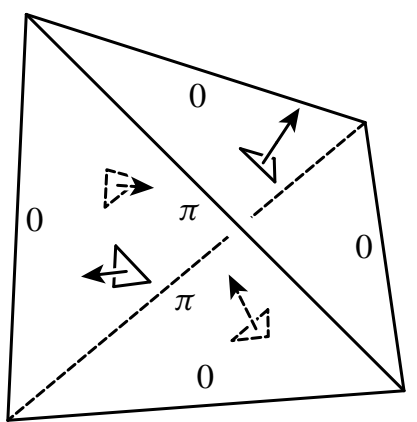

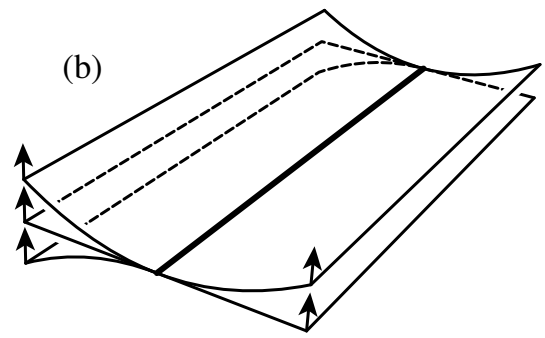

Figure 1: Conditions for a taut ideal triangulation

A taut ideal triangulation comes with a compatible taut angle structure, but not every taut angle structure arises from a taut structure. Examples of this are given in Section 4.

Agol [1] recently introduced extra structure on a taut ideal triangulation, which he terms veering, using a local condition. We define the notion of veering for any triangulation with a taut angle structure, and show that this new definition agrees with Agol's for taut ideal triangulations.

Let $\Delta^{3}$ be the standard 3-simplex with a chosen orientation. Suppose the edges of $\Delta^{3}$ are labelled by $e, e^{\prime}$ and $e^{\prime \prime}$, such that opposite edges have the same label and all three labels occur. Then the cyclic order of $e, e^{\prime}$ and $e^{\prime \prime}$ viewed from each vertex depends only on the orientation of the 3-simplex, ie is independent of the choice of vertex. It follows that, up to orientation preserving symmetries, there are two possible labellings, and we fix one of these labellings as shown in Figure 2. 
Definition 1.3 (Veering structure) A veering tetrahedron is an oriented angle-taut tetrahedron, where each edge with angle 0 is coloured either red or blue (drawn dotted and dashed respectively), such that the cyclic order of the edges at each vertex takes the $\pi$ angle edge to a blue edge to a red edge. This is shown in Figure 2. We refer to the red edges as right-veering and the blue edges as left-veering. Colours assigned to the $\pi$ angle edges are irrelevant to the definition of a veering tetrahedron. A triangulation $\mathcal{T}$ with a taut angle structure is a veering triangulation of $M$ if there is a colour assigned to each edge in the triangulation so that every tetrahedron is veering. This will also be called a veering structure on $M$.

Note that in a veering triangulation there are three different possible colourings of each tetrahedron: both $\pi$ angles red, both $\pi$ angles blue, and one of each colour.

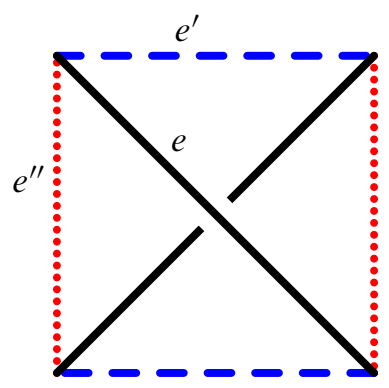

Figure 2: The canonical picture of a veering tetrahedron. The 0 angles are at the four sides of the square, and the $\pi$ angles are the diagonals. We indicate the veering directions on the 0 angle edges of a tetrahedron by colouring the edges. Note that this picture depends on a choice of orientation for the tetrahedron.

In this paper, angle-taut tetrahedra will generally be drawn flattened onto the page as in Figure 2, so that every tetrahedron appears as a quadrilateral with two diagonals, the four boundary edges all having angle 0 , and the two diagonals having angle $\pi$.

In Section 2, we will interpret the definitions in terms of normal surface theory, and give a proof of the following result.

Proposition 1.4 For a taut ideal triangulation, Definition 1.3 is equivalent to the definition of a veering taut triangulation given by Agol [1].

The main result of this paper is the following, a proof of which is given in Section 3. Our more general notion of veering triangulations was motivated by this result, which does not depend on the existence of a taut structure on $M$. 
Theorem 1.5 Veering triangulations of 3-manifolds admit strict angle structures.

In particular, using results of Casson and Thurston, it follows that any 3-manifold with a veering triangulation admits a complete hyperbolic structure.

Question 1.6 Can all veering triangulations be realised as ideal hyperbolic triangulations in which all tetrahedra are positively oriented?

Veering triangulations seem to be very special (see Remark 4.3 for some data from the SnapPea census), but many manifolds have them. Agol [1] proves that if we take any pseudo-Anosov mapping torus and puncture the surface along the singular points of the invariant measured foliations, then the mapping torus with the restricted monodromy has a layered triangulation with compatible veering and taut structures (see [1] for the sense of compatibility here). A layered triangulation is obtained by stacking tetrahedra on a triangulation of a surface. For instance, the canonical triangulations of once punctured torus bundles are all layered, taut and veering. Agol points out that the definition of veering does not depend on the triangulation being layered, and asks whether there is a veering taut triangulation which is not layered. In Section 4 we give such an example.

Question 1.7 In addition to layering, what other ways are there to generate veering triangulations?

This work was supported by Australian Research Council grant DP1095760.

\section{Definitions}

\subsection{Ideal triangulation}

Let $M$ be a topologically finite 3-manifold, ie the interior of a compact 3-manifold. An ideal triangulation $\mathcal{T}$ of $M$ consists of a pairwise disjoint union of standard Euclidean 3-simplices, $\widetilde{\Delta}=\bigcup_{k=1}^{n} \widetilde{\Delta}_{k}$, together with a collection $\Phi$ of Euclidean isometries between the 2 -simplices in $\widetilde{\Delta}$, called face pairings, such that $M \cong\left(\widetilde{\Delta} \backslash \widetilde{\Delta}^{(0)}\right) / \Phi$. The simplices in $M$ may be singular. It is well-known that every noncompact, topologically finite 3-manifold admits an ideal triangulation. We will assume throughout that $M$ as above is imbued with a fixed triangulation $\mathcal{T}$. We will also assume that $M$ is oriented and that all 3-simplices in $M$ are coherently oriented.

\subsection{Quadrilateral types}

Let $\Delta^{3}$ be the standard 3-simplex with a chosen orientation. As stated in the introduction, the edges of $\Delta^{3}$ are labelled by $e, e^{\prime}$ and $e^{\prime \prime}$, such that opposite edges have the 
same label and all labels occur. This determines the same cyclic order of $e, e^{\prime}$ and $e^{\prime \prime}$ viewed from each vertex. Our convention is illustrated in Figure 2.

Each pair of opposite edges corresponds to a normal isotopy class of quadrilateral discs in $\Delta^{3}$, disjoint from the pair of edges. We call such an isotopy class a normal quadrilateral type. There is a natural cyclic order on the set of normal quadrilateral types induced by the cyclic order on the edges from a vertex, and this order is preserved by all orientation preserving symmetries of $\Delta^{3}$. The particular cyclic order chosen corresponds to the 3-cycle $\left(q q^{\prime} q^{\prime \prime}\right)$, where $q^{(k)}$ is dual to $e^{(k)}$.

Let $M^{(k)}$ be the set of all $k$-simplices in $M$. If $\sigma \in M^{(3)}$, then there is an orientation preserving map $\Delta^{3} \rightarrow \sigma$ taking the $k$-simplices in $\Delta^{3}$ to elements of $M^{(k)}$, and which is a bijection between the sets of normal quadrilateral types. This map induces a cyclic order of the normal quadrilateral types in $\sigma$, and we denote the corresponding 3 -cycle $\tau_{\sigma}$. It follows from the above remarks that this order is independent of the choice of the map.

Let $\square$ denote the set of all normal quadrilateral types in $M$. Define the permutation $\tau$ of $\square$ by

$$
\tau=\prod_{\sigma \in M^{3}} \tau_{\sigma} .
$$

See Figure 5 for an illustration of the action of $\tau$ on the three quadrilateral types in a tetrahedron.

If $e \in M^{(1)}$ is any edge, then there is a sequence $\left(q_{n_{1}}, \ldots, q_{n_{k}}\right)$ of normal quadrilateral types facing $e$, which consists of all normal quadrilateral types dual to $e$ listed in sequence as one travels around $e$. Then $k$ equals the degree of $e$, and a normal quadrilateral type may appear at most twice in the sequence. This sequence is welldefined up to cyclic permutations and reversing the order.

\subsection{Angle structures}

Definition 2.1 (Generalised angle structure) A function $\alpha: \square \rightarrow \mathbb{R}$ is called a generalised angle structure on $M$ if it satisfies the following two properties.

(1) If $\sigma^{3} \in M^{(3)}$ and $q$ is a normal quadrilateral type supported by it, then

$$
\alpha(q)+\alpha(\tau q)+\alpha\left(\tau^{2} q\right)=\pi .
$$

(2) If $e \in M^{(1)}$ is any edge and $\left(q_{n_{1}}, \ldots, q_{n_{k}}\right)$ is its normal quadrilateral type sequence, then

$$
\sum_{i=1}^{k} \alpha\left(q_{n_{i}}\right)=2 \pi
$$


Dually, one can regard $\alpha$ as assigning angles $\alpha(q)$ to the two edges opposite $q$ in the tetrahedron containing $q$.

A generalised angle structure is called a taut angle structure on $M$ if its image is contained in $\{0, \pi\}$, a semiangle structure on $M$ if its image is contained in $[0, \pi]$, and a strict angle structure on $M$ if its image is contained in $(0, \pi)$.

\subsection{Agol's definition}

Throughout this subsection, suppose $M$ has a veering triangulation with underlying taut angle structure $\alpha: \square \rightarrow\{0, \pi\}$. The conventions regarding orientations and normal quadrilateral types immediately imply the following fact (compare Figure 5).

Lemma 2.2 Suppose $\sigma$ is a tetrahedron in $M$. Let $q$ be the quadrilateral type dual to the edges with label $\pi$. Then the edges dual to $\tau(q)$ are left-veering (blue) and the edges dual to $\tau^{2}(q)$ are right-veering (red).

If $e \in M^{(1)}$ is any edge and $\left(q_{n_{1}}, \ldots, q_{n_{k}}\right)$ is its normal quadrilateral type sequence, then there are precisely two normal quadrilateral types in this sequence on which $\alpha$ takes the value $\pi$. These separate the sequence into two subsequences of consecutive 0 -angle quadrilateral types, which we call the sides of $e$. Their lengths are called the one-sided degrees of $e$.

Lemma 2.3 Each one-sided degree of $e$ is at least one.

Proof At each vertex of a veering tetrahedron there is a cyclic order of the three edges of the tetrahedron, taking the $\pi$-edge to the left-veering (blue) edge to the right-veering (red) edge. Notice that the order is the same at every vertex. This implies that the two normal quadrilateral types dual to the edge $e$ having angle $\pi$ cannot be adjacent in the normal quadrilateral type sequence of $e$, since otherwise there would be a conflict in the colouring. See Figure 3.

Corollary 2.4 The degree of each edge is at least four.

We now show that for a taut ideal triangulation, Definition 1.3 is equivalent to the definition of a veering taut triangulation given by Agol [1, Definition 4.1]. According to that definition, one needs to check two conditions for each edge in the triangulation. The second condition is already given by Lemma 2.3. The first is verified by the following lemma. See [1] for further context of the terms involved in its statement. 


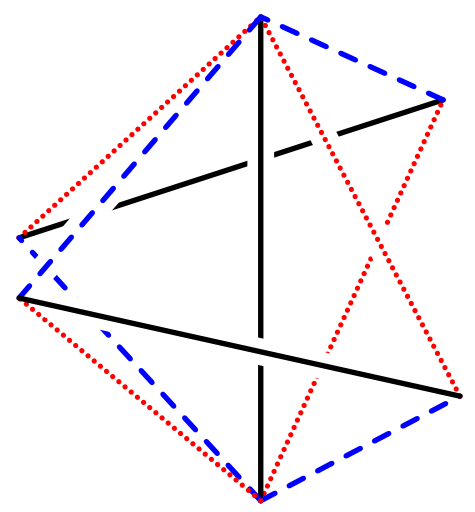

Figure 3: The one-sided degree in a veering taut triangulation is at least one.

Lemma 2.5 Suppose that the triangulation of $M$ is taut and veering, and has a taut angle structure compatible with both. Let e be a right (resp. left)-veering edge in $M$. Consider the sequence of oriented triangles incident to one side of $e$, and the vertices of these triangles opposite $e$. If we order the vertices moving "upwards" according to the coorientation of the triangles, they are also ordered from left to right (resp. right to left) as viewed from $e$. Similarly for the other side of $e$.

See Figure 4(a) for a picture of triangles incident to a right veering edge, veering to the right as the triangles are ordered approaching the reader.
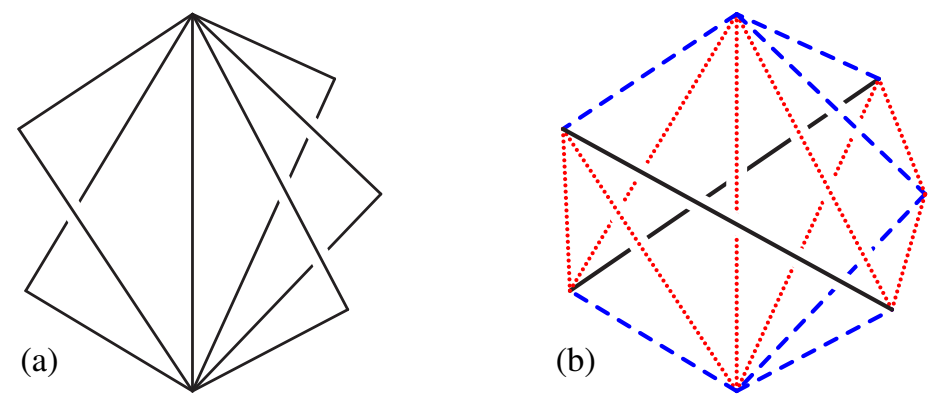

Figure 4: The triangles adjacent to a right-veering edge, and veering directions on the link of a right-veering edge

Proof The result is a consequence of stacking copies of tetrahedra coloured as in Definition 1.3 at $e$ so that the colours match. We have already observed the pattern on the two tetrahedra with label $\pi$ at $e$ in Figure 3. The result now follows by induction on the number of tetrahedra abutting $e$ by considering the cases of whether $e$ is leftveering (blue) or right-veering (red). See Figure 4(b) for an example. 
Proof of Proposition 1.4 Lemma 2.3 and Lemma 2.5 immediately imply the forward direction of Proposition 1.4. For the reverse direction: a taut triangulation satisfying the conclusion of Lemma 2.5 has veering directions on the 0 angle edges of each tetrahedron matching the colouring given by Definition 1.3. To see this, observe that each of the 0 angle edges in Figure 2 has a pair of incident triangles, determining the veering direction.

Remark 2.6 Lemma 2.2 points to a further generalisation of veering triangulations, separated from an underlying taut angle structure. It consists merely of a choice of normal quadrilateral type $q$ in each oriented tetrahedron subject to compatibility of edge veering directions, where for each tetrahedron the left and right-veering edges are defined to be those dual to $\tau(q)$ and $\tau^{2}(q)$ respectively. In the current sense of veering, for each tetrahedron the choice of $q$ satisfies $\alpha(q)=\pi$.

\section{Veering triangulations and angle structures}

The main result of this paper is the following:

Theorem 1.5 Veering triangulations of 3-manifolds admit strict angle structures.

Work of Kang and Rubinstein [9] and Luo and Tillmann [11] links the existence of angle structures to the normal surface theory of $M$ using duality principles from linear programming. The normal surface solution space $C(M ; \mathcal{T})$ is a vector subspace of $\mathbb{R}^{7 n}$, where $n$ is the number of tetrahedra in $\mathcal{T}$, consisting of vectors satisfying the compatibility equations of normal surface theory. The coordinates of $x \in \mathbb{R}^{7 n}$ represent weights of the four normal triangle types and the three normal quadrilateral types in each tetrahedron, and the compatibility equations state that normal triangles and quadrilaterals have to meet the 2-simplices of $\mathcal{T}$ with compatible weights.

A vector in $\mathbb{R}^{7 n}$ is called admissible if at most one quadrilateral coordinate from each tetrahedron is nonzero and all coordinates are nonnegative. An integral admissible element of $C(M ; \mathcal{T})$ corresponds to a unique embedded, closed normal surface in $M$ and vice versa. As a reference for other facts from normal surface theory, please consult Jaco and Oertel [7].

There is a linear function $\chi^{*}: C(M ; \mathcal{T}) \rightarrow \mathbb{R}$, which agrees with the Euler characteristic $\chi$ on embedded and immersed normal surfaces. If $\mathcal{T}$ admits a generalised angle structure $\alpha$, then the formal Euler characteristic $\chi^{*}$ can be computed by

$$
2 \pi \chi^{*}(x)=\sum_{q}-2 \alpha(q) x_{q},
$$

where $x_{q}$ is the normal coordinate of the normal quadrilateral type $q$. 
Since a taut angle structure has image $\{0, \pi\}$, we have the following simple application of Theorems 1 and 3 of [11].

Corollary 3.1 If $M$ has a taut angle structure, then the following are equivalent:

(1) $M$ admits a strict angle structure.

(2) For all $x \in C(M ; \mathcal{T})$ with all quadrilateral coordinates nonnegative and at least one quadrilateral coordinate positive, $\chi^{*}(x)<0$.

(3) There is no $x \in C(M ; \mathcal{T})$ with all quadrilateral coordinates nonnegative and at least one quadrilateral coordinate positive and $\chi^{*}(x)=0$.

The quadrilateral coordinates in the solutions to the compatibility equations satisfy the so-called $Q$-matching equations due to Tollefson [12]. Given the sequence $\left(q_{n_{1}}, \ldots, q_{n_{k}}\right)$ of normal quadrilateral types facing an edge $e$, one associates a sign $\varepsilon(q) \in\{ \pm 1\}$ to each element $q$ in the sequence $\left(\tau\left(q_{n_{1}}\right), \tau^{2}\left(q_{n_{1}}\right), \ldots, \tau\left(q_{n_{k}}\right), \tau^{2}\left(q_{n_{k}}\right)\right)$ of quadrilateral types incident with $e$. If a normal quadrilateral type appears more than once in this sequence, it may have different signs. The $Q$-matching equation associated to $e$ is

$$
\sum \varepsilon(q) x_{q}=0
$$

where the sum is taken over the elements of $\left(\tau\left(q_{n_{1}}\right), \tau^{2}\left(q_{n_{1}}\right), \ldots, \tau\left(q_{n_{k}}\right), \tau^{2}\left(q_{n_{k}}\right)\right)$. In the situation of a veering triangulation, the signs are as given in Figure 5. This suggests another alternate interpretation of the colouring on a veering tetrahedron in terms of the signs of the quadrilateral type dual to the $\pi$ edges.

Definition 3.2 For a triangulation with a taut angle structure the three quadrilateral types within each tetrahedron fall into two classes: (i) there are two kinds of vertical quadrilateral types (denoted vertical-1 and vertical-2) which have two 0 angle and two $\pi$ angle corners, and (ii) one kind of horizontal quadrilateral type, which has four 0 angle corners. If $q$ is the horizontal quadrilateral type in a tetrahedron, then $\tau(q)$ and $\tau^{2}(q)$ are the vertical-1 and vertical-2 quadrilateral types respectively. See Figure 5.

Note that a positive coordinate corresponding to a horizontal quadrilateral type contributes negatively to $\chi^{*}(x)$, whereas those corresponding to vertical quadrilateral types contribute 0 . We remark that the set of solutions to the $Q$-matching equations also includes the so-called spun-normal surfaces, which do not appear as solutions in $C(M ; \mathcal{T})$. Thus nonexistence of solutions to the $Q$-matching equations under the conditions of Corollary 3.1(3) implies (but is not implied by) nonexistence of $x \in C(M ; \mathcal{T})$ with those conditions, and we have the following: 


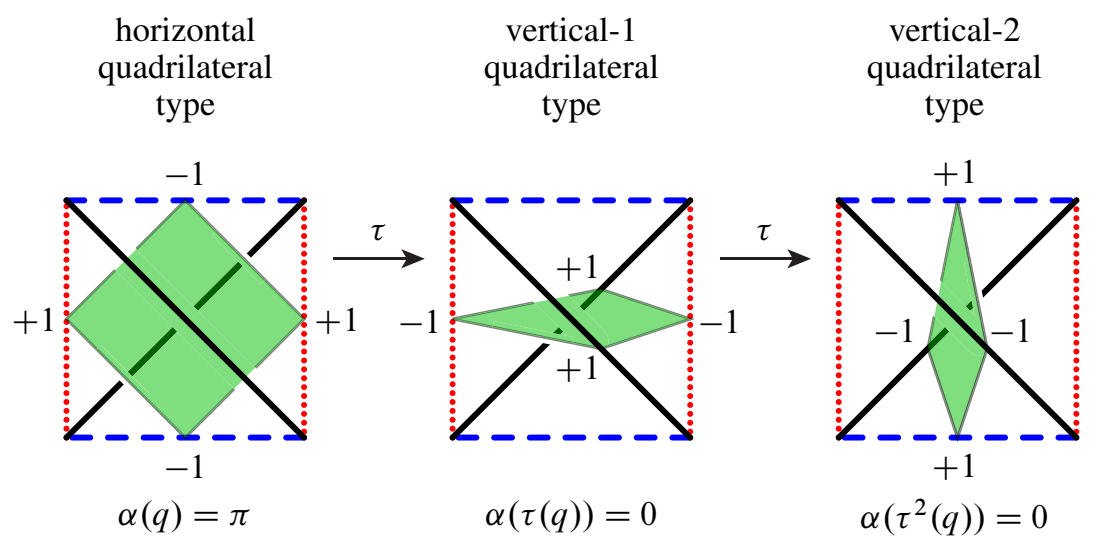

Figure 5: The one horizontal and two vertical quadrilateral types in an angletaut tetrahedron, with associated signs in the $Q$-matching equations

Lemma 3.3 A triangulation with a taut angle structure admits a strict angle structure if it admits no nonnegative solution to the $Q$-matching equations with at least one quadrilateral coordinate positive and for which every nonzero quadrilateral type is vertical.

Hence Theorem 1.5 follows from the following:

Proposition 3.4 If $M$ has a veering triangulation, then there is no nonnegative solution to the $Q$-matching equations with at least one quadrilateral coordinate positive and for which every nonzero quadrilateral is vertical.

Proof By way of contradiction, suppose there is a nonnegative solution $x$ to the $Q$-matching equations with at least one quadrilateral coordinate positive and for which every nonzero quadrilateral type is vertical.

Suppose $q$ is a vertical-1 quadrilateral type with $x_{q}>0$, supported by the tetrahedron $\sigma$. Then the signs associated to its corners on the red edges of $\sigma$ are both negative, and hence its contribution to the respective $Q$-matching equations is negative. This cannot be compensated for by vertical-2 quadrilateral types, since their positive signs are on blue edges. It follows that it must be compensated for by vertical-1 quadrilateral types.

This implies that the total negative contribution of all nonzero vertical-1 quadrilateral types to the sum of all $Q$-matching equations of the red edges equals the total positive contribution of all vertical-1 quadrilateral types to the sum of all $Q$-matching equations of the red edges. Hence a vertical-1 quadrilateral type with nonzero coordinate must have all four corners on red edges; similarly for vertical-2 quadrilateral types and blue 
edges. So the $Q$-matching equations restricted to red edges and vertical-1 quadrilateral types are independent of the $Q$-matching equations restricted to blue edges and vertical-2 quadrilateral types. Therefore we may assume that $x_{q}=0$ for each vertical-2 quadrilateral type. This implies that the solution $x$ is admissible. Since $x$ is a convex linear combination of the so-called admissible vertex solutions, we may assume that $x$ is an admissible vertex solution, and may hence choose a connected, oriented spunnormal surface $S$ in the projective normal isotopy class defined by $x$. The spun-normal surface $S$ may be noncompact or closed (normal). Since $S$ is oriented, we can choose a transverse orientation to $S$.

The argument in the previous two paragraphs goes through verbatim if we interchange "vertical-1" with "vertical-2" and "red" with "blue", as does the rest of the proof (also interchanging " +1 " with " -1 ").

We now analyse how the quadrilateral discs of the surface $S$ sit in the triangulation. First notice that each quadrilateral disc has all of its corners on red edges. The two corners with sign +1 inherit angle $\pi$, and the corners with sign -1 inherit angle 0 . Since $S$ is embedded and the sum of angles around any edge equals $2 \pi$, it follows that at any vertex in the induced cell structure of $S$, there are at most four quadrilateral discs.

The next claim pertains to the tetrahedra with four edges of the same colour. As noted above, all tetrahedra containing quadrilaterals of $S$ have this property. We use the notion of the sides of an edge, as introduced after Lemma 2.2.

Property $(*)$ Suppose edge $e$ is red, and has the sequence $\left(q_{n_{1}}, \ldots q_{n_{k}}\right)$ of quadrilateral types facing it, where $\alpha\left(q_{n_{1}}\right)=0$ and the tetrahedron $\sigma$ supporting $q_{n_{1}}$ has four red edges. Then $\alpha\left(q_{n_{2}}\right)=\alpha\left(q_{n_{k}}\right)=\pi$, which implies that the subsequence $\left(q_{n_{1}}\right)$ is a side of $e$ (and so the associated one-sided degree is 1).

We now prove Property $(*)$. The tetrahedron $\sigma$ has both $\pi$ angles right-veering, and has angle 0 at the edge $e$. See Figure 6. First consider the tetrahedron $\sigma^{\prime}$ that is glued to the triangle $\Delta$, which is the triangle on the front of $\sigma$ that is incident to $e$. Since $\sigma^{\prime}$ is veering and its face $\Delta$ has two right-veering (red) edges, it follows that the edge of $\sigma^{\prime}$ opposite the left-veering (blue) edge of $\Delta$ is also left-veering (blue), since both colours appear at each vertex of each tetrahedron. The cyclic order " $\pi \rightarrow$ blue $\rightarrow$ red" at the vertex of $\sigma^{\prime}$ incident with the two right-veering (red) edges of $\Delta$ now implies that $\sigma^{\prime}$ has angle $\pi$ at $e$, ie $\alpha\left(q_{n_{2}}\right)=\pi$. A similar argument shows that the same is true for the tetrahedron glued to the triangle on the back of $\sigma$ that is incident to $e$, giving $\alpha\left(q_{n_{k}}\right)=\pi$. It follows that $\left(q_{n_{1}}\right)$ is a side of $e$. See the right diagram of Figure 6. 

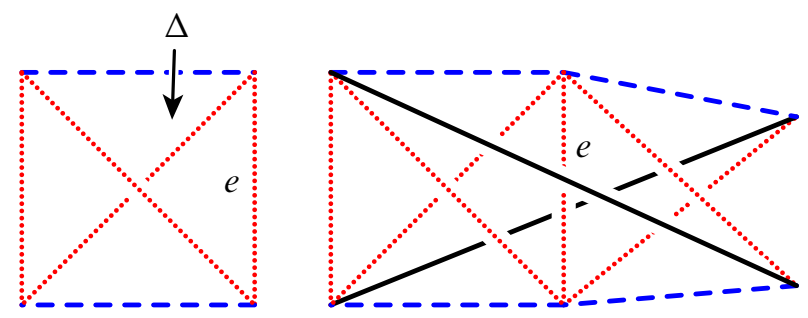

Figure 6: On the left: a tetrahedron with both $\pi$ angles right-veering (red). On the right: this choice of veering implies that this tetrahedron contributes the only 0 angle on its side of $e$.

Property $(*)$ places severe restrictions on the cell structure of $S$. If a quadrilateral disc meets an edge $e$ with one of its -1 corners, then it is contained in a tetrahedron corresponding to the $\left(q_{n_{1}}\right)$ side of $e$. Thus, at each vertex in the induced cell structure of $S$, either precisely two quadrilaterals or precisely four quadrilaterals meet. Moreover, the former is the case if $\operatorname{deg}(e) \geq 5$, and the latter can only happen if $\operatorname{deg}(e)=4$. In addition, each quadrilateral in $S$ meets quadrilaterals (rather than triangles) along at least two of its edges.

We will now use the transverse orientation and the natural geometry of quadrilaterals to start exploring the subsurface of $S$ made up of quadrilateral discs. See Figure 7. Suppose we start on $q_{0}$. We set our compass so that one of the positive corners is north, then the other is south and the negative corners are east and west. As we walk from $q_{0}$ to another quadrilateral disc, we parallel transport our compass, and do not keep track of whether we visited this disc previously. Given an arbitrary quadrilateral disc $q$, Property (*) implies that we can always find a quadrilateral disc in $S$ meeting $q$ along at least one of its two sides incident to a -1 corner. For $q_{0}$ this means that it meets a quadrilateral disc along either the northeast or southeast edge. This is true for any quadrilateral disc we have parallel transported our compass to.

Suppose we cannot walk from $q_{0}$ indefinitely in the northeasterly direction. Then after travelling in the northeasterly direction across finitely many quadrilateral discs, we reach a quadrilateral disc $q_{k}$, which meets a normal triangle along its northeast side. Since the eastern corner has a $-1, q_{k}$ must meet a quadrilateral disc along its southeast side.

Claim We can walk from $q_{k}$ indefinitely in the southeasterly direction.

The proof is by contradiction and sketched in Figure 7. Suppose to the contrary that after traversing discs $q_{k+1}, \ldots, q_{n-1}$, we reach a disc $q_{n}$ which does not meet another quadrilateral disc along its southeast side. Then it must meet another along its northeast 


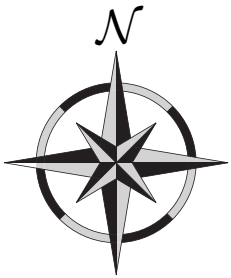

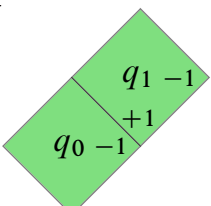

(a)

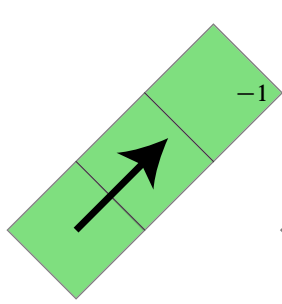

(b)

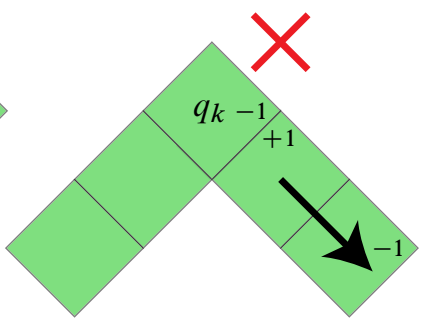

(c)
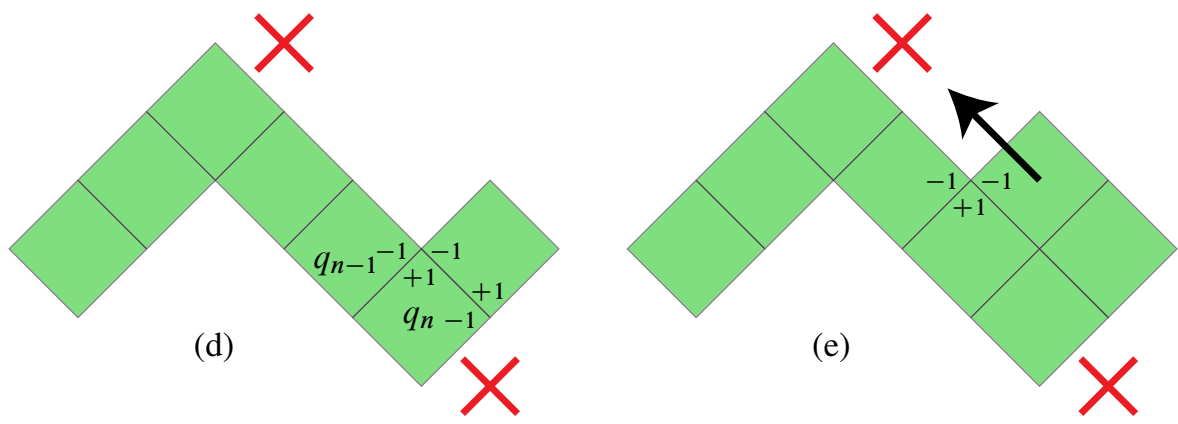

Figure 7: Traversing through quadrilateral discs

side in order to cancel its eastern -1 corner. But then the edge at its northern +1 corner is incident with three quadrilaterals, and hence must be incident with four. This implies that this edge is of degree four. But then $q_{n-1}$ is glued along its northeast edge to another quadrilateral. This now propagates all the way to $q_{k}$, giving a contradiction. This proves the claim.

In all cases then, there is a quadrilateral disc $q_{0}$ in $S$, from which one can walk indefinitely across quadrilaterals. Let $a$ be the normal arc in the boundary of $q_{0}$ that we walk across (as shown in Figure 8). We may assume that the transverse orientation of $S$

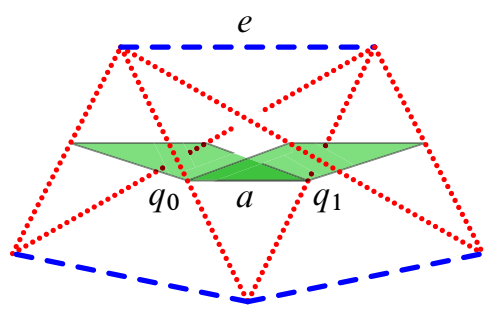

Figure 8: The dual edge remains the same as we walk across quadrilateral discs. 
induced on $a$ points not to the ideal vertex of the triangle containing $a$, but to the ideal edge $e$ which $a$ does not meet. Then $q_{1}$ again has its transverse orientation pointing towards $e$ and is also dual to it. It follows inductively that all quadrilateral discs in the indefinite path are dual to $e$, and that all quadrilateral types dual to $e$ appear in $S$ and are therefore all vertical. But then the total angle sum at $e$ is zero, contradicting the fact that $M$ has a taut angle structure.

Remark 3.5 If the given manifold with veering triangulation is known to be hyperbolic, then we can alter the proof in the following way: Since the surface $S$ is embedded, we have $\chi(S)=\chi^{*}(x)=0$, and since it is orientable and connected it must be a torus or a spun-normal annulus. Theorem 2.6 of [9] then tells us that this torus must be nonboundary parallel and essential, leading to a contradiction to the assumption that the manifold is hyperbolic. If there is an annulus, there are two cases. If the annulus is boundary parallel, one can use this surface as a barrier (as in Jaco and Rubinstein [8]) and find a normal torus which is topologically but not normally parallel to the boundary, giving a contradiction as in [9]. If there is an essential annulus then the manifold is again not hyperbolic.

\section{A veering taut triangulation that is not layered}

Agol's construction [1] gives us the existence of many veering taut triangulations, but they are all layered triangulations. In this section we give an example of a veering taut triangulation that is not layered. In fact, the manifold does not fibre over the circle. We also list some examples of veering triangulations that do not fibre over the circle and are not taut triangulations.

The main example is the manifold s227 from the SnapPea census [13], with the triangulation as given in the census. See Figure 9. One can show that the manifold s227 is not fibred by applying a method due to Brown [2] to compute the BNS invariant. More specifically, the fundamental group of the manifold is isomorphic to

$$
\Gamma=\left\langle a, b \mid a^{4} b^{2} a^{-1} b^{-1} a^{-1} b^{2} a^{-1} b^{-1} a^{-1} b^{2}=1\right\rangle,
$$

and hence has two generators and one relator, with abelianisation isomorphic to $\mathbb{Z} \oplus \mathbb{Z}_{4}$. An application of the algorithm described in [2, Theorem 4.2] verifies that there is no epimorphism $\Gamma \rightarrow \mathbb{Z}$ with finitely generated kernel.

Remark 4.1 This example was found by searching the SnapPea census using the Regina software package by Ben Burton [3], together with a listing of SnapPea census manifolds with data on whether or not they fibre compiled by Nathan Dunfield [5]. 

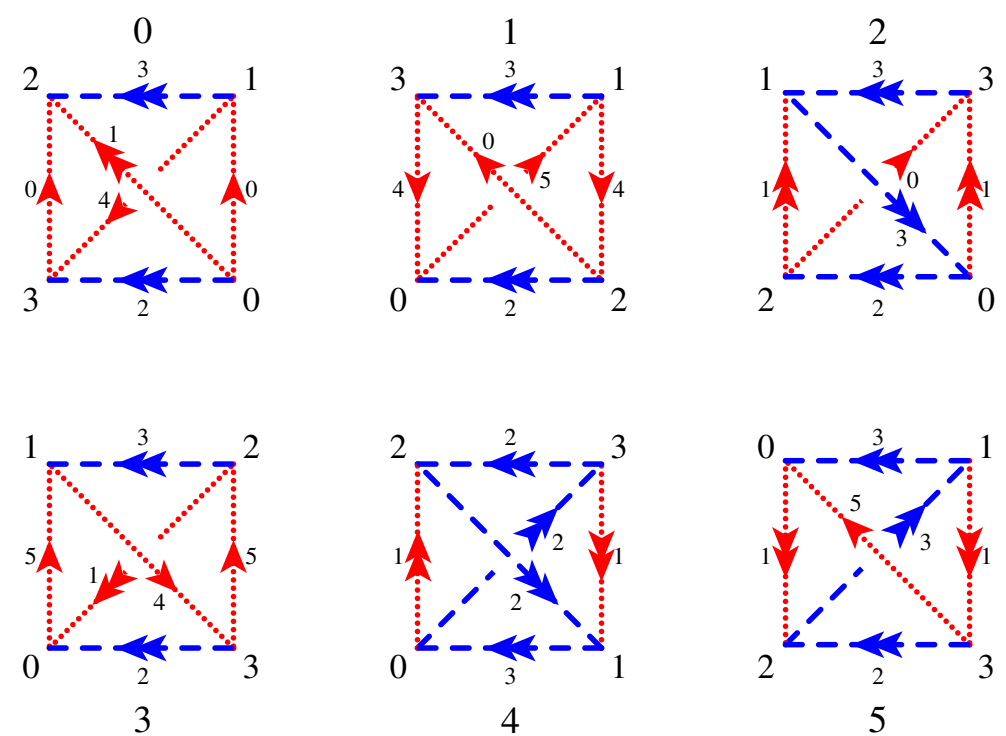

Figure 9: The triangulation s227 from the SnapPea census, with the veering taut structure indicated. There are six tetrahedra labelled from 0 to 5 and six edges, also labelled from 0 to 5 . Single arrows refer to degree 4 edges and double arrows degree 8 edges. We have drawn the tetrahedra so that they are taut, flattened onto the page as in Figure 2, with consistent coorientation of the triangles in the direction out of the page towards the reader. Moreover, the orientation of each tetrahedron agrees with the vertex sequence $0,1,2,3$.

There is no guarantee that this is the "smallest" example of a nonfibred veering taut triangulation, since we only checked the triangulation of each manifold as it appears in the census. In addition we have not checked for layering directly; there are a number of triangulations in the census with taut angle structures that are both taut and veering, and it is likely that some of these examples are not layered even if the manifold fibres.

Remark 4.2 Examples of veering triangulations of manifolds that do not fibre over the circle but that are not taut triangulations were found by a similar method. They are s438, s772, s773, s779, v3128, v3243, v3244, v3377 and v3526, again with the triangulations as given in the SnapPea census and taut angle structure as given in Table 1. These manifolds do not fibre either by the BNS invariant again (as calculated by Dunfield), or by Button [4].

Remark 4.3 There are 4,815 orientable triangulations in the SnapPea census, which lists triangulations with up to 7 tetrahedra. On those triangulations we calculate a total of 13,599 taut angle structures, of which 10,204 have a compatible taut structure 
and 158 have a compatible veering structure. There are 125 taut angle structures that are both taut and veering; all but one (s227) of the associated manifolds have some triangulation that is layered, according to Dunfield's data. Thus, we have at least 34 veering structures not coming from Agol's construction.

Remark 4.4 We have also attempted to find veering triangulations by randomised retriangulation of the SnapPea census triangulations. Within the $\leq 5$ tetrahedron census (a total of 301 orientable manifolds) we have so far found 56 distinct veering taut angle structures on 54 distinct triangulations of 53 manifolds. The canonical triangulations of the manifolds $\mathrm{m} 135$ and $\mathrm{m} 136$ each have two veering taut angle structures, while the manifold $\mathrm{m} 140$ has two distinct triangulations (neither of which is canonical), each with a veering taut angle structure. Of the 54 triangulations, 33 are canonical and the remaining 21 are not. We think that it is likely that there are other veering triangulations on these manifolds that our search did not find. Every veering triangulation we have found so far seems to be geometric: SnapPea claims to find a complete hyperbolic structure for the manifold for which every ideal tetrahedron is positively oriented in $\mathbb{H}^{3}$.

\begin{tabular}{|r|ccccccc|}
\hline Tetrahedron & 0 & 1 & 2 & 3 & 4 & 5 & 6 \\
\hline s227 & $e_{02}, e_{13}$ & $e_{01}, e_{23}$ & $e_{01}, e_{23}$ & $e_{02}, e_{13}$ & $e_{03}, e_{12}$ & $e_{03}, e_{12}$ & - \\
s438 & $e_{02}, e_{13}$ & $e_{02}, e_{13}$ & $e_{02}, e_{13}$ & $e_{02}, e_{13}$ & $e_{02}, e_{13}$ & $e_{01}, e_{23}$ & - \\
s772 & $e_{02}, e_{13}$ & $e_{01}, e_{23}$ & $e_{01}, e_{23}$ & $e_{01}, e_{23}$ & $e_{01}, e_{23}$ & $e_{02}, e_{13}$ & - \\
s773 & $e_{02}, e_{13}$ & $e_{01}, e_{23}$ & $e_{01}, e_{23}$ & $e_{01}, e_{23}$ & $e_{01}, e_{23}$ & $e_{02}, e_{13}$ & - \\
s779 & $e_{02}, e_{13}$ & $e_{01}, e_{23}$ & $e_{01}, e_{23}$ & $e_{01}, e_{23}$ & $e_{01}, e_{23}$ & $e_{02}, e_{13}$ & - \\
v3128 & $e_{02}, e_{13}$ & $e_{02}, e_{13}$ & $e_{02}, e_{13}$ & $e_{02}, e_{13}$ & $e_{02}, e_{13}$ & $e_{02}, e_{13}$ & $e_{01}, e_{23}$ \\
v3243 & $e_{01}, e_{23}$ & $e_{02}, e_{13}$ & $e_{02}, e_{13}$ & $e_{02}, e_{13}$ & $e_{02}, e_{13}$ & $e_{01}, e_{23}$ & $e_{02}, e_{13}$ \\
v3244 & $e_{01}, e_{23}$ & $e_{03}, e_{12}$ & $e_{03}, e_{12}$ & $e_{03}, e_{12}$ & $e_{03}, e_{12}$ & $e_{01}, e_{23}$ & $e_{03}, e_{12}$ \\
v3377 & $e_{02}, e_{13}$ & $e_{02}, e_{13}$ & $e_{02}, e_{13}$ & $e_{02}, e_{13}$ & $e_{02}, e_{13}$ & $e_{01}, e_{23}$ & $e_{02}, e_{13}$ \\
v3526 & $e_{01}, e_{23}$ & $e_{03}, e_{12}$ & $e_{03}, e_{12}$ & $e_{02}, e_{13}$ & $e_{02}, e_{13}$ & $e_{03}, e_{12}$ & $e_{01}, e_{23}$ \\
\hline
\end{tabular}

Table 1: Taut angle structures corresponding to veering triangulations for manifolds that do not fibre. The edges with angle $\pi$ are listed. The edge subscripts are the vertex numbers as given in the SnapPea census.

\section{References}

[1] I Agol, Ideal triangulations of pseudo-Anosov mapping tori arXiv:1008.1606v2

[2] K S Brown, Trees, valuations, and the Bieri-Neumann-Strebel invariant, Invent. Math. 90 (1987) 479-504 MR914847 
[3] B A Burton, Regina: Normal surface and 3-manifold topology software (1999-2009) Available at http://regina.sourceforge.net/

[4] J O Button, Fibred and virtually fibred hyperbolic 3-manifolds in the censuses, Experiment. Math. 14 (2005) 231-255 MR2169525

[5] N Dunfield, Which cusped census manifolds fiber? (2010) Available at http:// dunfield.info/snappea/tables/mflds_which_fiber

[6] F Guéritaud, On canonical triangulations of once-punctured torus bundles and twobridge link complements, Geom. Topol. 10 (2006) 1239-1284 MR2255497 With an appendix by D Futer

[7] W Jaco, U Oertel, An algorithm to decide if a 3-manifold is a Haken manifold, Topology 23 (1984) 195-209 MR744850

[8] W Jaco, J H Rubinstein, 0-efficient triangulations of 3-manifolds, J. Differential Geom. 65 (2003) 61-168 MR2057531

[9] E Kang, J H Rubinstein, Ideal triangulations of 3-manifolds II; taut and angle structures, Algebr. Geom. Topol. 5 (2005) 1505-1533 MR2186107

[10] M Lackenby, Taut ideal triangulations of 3-manifolds, Geom. Topol. 4 (2000) 369395 MR1790190

[11] F Luo, S Tillmann, Angle structures and normal surfaces, Trans. Amer. Math. Soc. 360 (2008) 2849-2866 MR2379778

[12] JL Tollefson, Normal surface Q-theory, Pacific J. Math. 183 (1998) 359-374 MR1625962

[13] J R Weeks, SnapPea: A computer program for creating and studying hyperbolic 3manifolds Available at http://www.geometrygames.org/SnapPea/

CH, JHR, HS: Department of Mathematics and Statistics, The University of Melbourne Melbourne Parkville VIC 3010, Australia

ST: School of Mathematics and Physics, The University of Queensland Brisbane QLD 4072, Australia

craigdh@unimelb.edu.au, H.Rubinstein@ms.unimelb.edu.au, segerman@unimelb.edu.au, tillmann@maths.uq.edu.au

http://www.ms. unimelb.edu.au/ cdh/,

http://www.ms.unimelb.edu.au/ rubin/, http://www.ms.unimelb.edu.au/ rsegerman/, http://www.maths.uq.edu.au/ tillmann/

Proposed: David Gabai

Seconded: Joan Birman, Colin Rourke
Received: 30 November 2010 Revised: 17 June 2011 